\section{Reply to C.L. Braal et al, H. Brauch et al, and M.P. Goetz et al}

We read the comments of Braal et al, ${ }^{1}$ Brauch et al, ${ }^{2}$ and Goetz et al ${ }^{3}$ about the CYPTAM study. ${ }^{4}$ Concerns were expressed about our sample size calculation. As described, sample size was calculated based on the studies by Goetz et al ${ }^{5}$ and Gonzalez-Santiago et al, ${ }^{6}$ in which hazard ratios (HRs) of 1.86 and 2.82, respectively, were reported. The assumed HR in a sample size calculation is determined by which effect size is considered to be clinically relevant. Certainly, one can debate what is clinically relevant, but we considered a doubling of the event rate of clinical relevance. Consequently, it seems inappropriate to adapt the HR in the sample size calculation for duration of tamoxifen exposure because it is not related to clinical relevance. For clarity, in our sample size calculation, the expected number of events at the 2-year follow-up were considered, and our assumptions were met (expected survival $v$ survival rate, 93\% v 94\%). Also, when comparing ultrarapid metabolizers plus extensive metabolizers (EMs) versus heterozygous EMs plus intermediate metabolizers plus poor metabolizers (PMs), we found 20 events in both groups at the 2.5-year follow-up.

We disagree with Brauch et al ${ }^{2}$ that the meta-analysis of Province et al ${ }^{7}$ reporting a lower HR of 1.25 could be informative for sample size calculation. This study was criticized for reasons of multiplicity, lack of study protocol, or statistical analysis plan and was designated as a negative study. ${ }^{8}$ Furthermore, an HR of 1.25 for CYP2D6 PMs would lead to approximately 1,000 patients needing to be genotyped to prevent one event, which may indicate lack of clinic utility. Nevertheless, it was mentioned in our Discussion that an effect with an HR of less than 2.0 cannot be excluded.

Our population consisted of patients with early breast cancer treated with adjuvant tamoxifen. Patients received prior adjuvant chemotherapy (61.5\%) or trastuzumab (8.7\%). Goetz et al ${ }^{5}$ stipulated that studies should be performed in a proper population. There can be no debate that results of a study are valid only in populations with similar characteristics.

We agree, for example, that adjuvant chemotherapy reduces the hazard for events. Consequently, an association of CYP2D6 genotype and events will be more difficult to prove and requires a higher sample size in patients receiving adjuvant chemotherapy. More important, the effect of CYP2D6 genotyping in these patients will be reduced. Therefore, we agree with Goetz et al ${ }^{5}$ that the impact of CYP2D6 genotyping in patients receiving monotherapy tamoxifen remains unknown, simply because this was not our research question and population. Similarly, concerns are expressed about the switch to aromatase inhibitors (Als) in $66 \%$ of the patients. In the last 10 years, guidelines have recommended a switch to an Al after 2 to 3 years of tamoxifen treatment in postmenopausal women. Therefore, it is of no surprise that the majority of patients used tamoxifen for approximately 2.5 years. Having become the emerging standard of care, we believe it makes sense to study the role of CYP2D6 genotyping in this setting. Therefore, patients were censored at the time of tamoxifen discontinuation, and the potential effect modification by Al was avoided. However, when examining the complete relapse-free survival, including Al therapy, no association with CYP2D6 was also found. Taken all together, we reiterate that our results cannot be extrapolated to other tamoxifen regimens and/or patient populations. It is a strength that our study reflects common practice of mostly postmenopausal patients starting with tamoxifen and switching to an $\mathrm{Al}$ after 2 to 3 years of treatment, which may also implicate that the role of CYP2D6 genotyping is smaller than in regimens in which tamoxifen was given for 5 years.

Brauch et al ${ }^{2}$ also comment on the CYP2D6 phenotype stratification. After our analysis plan, we analyzed ultrarapid metabolizers plus EMs versus heterozygous EMs plus intermediate metabolizers plus PMs and found a $P$ value of .799 , without even a trend for significance. However, adding PMs as a subgroup did not alter our results $(P=.277)$.

Braal et al ${ }^{1}$ state that "the primary end point of this study was not to investigate the relationship between endoxifen concentrations and clinical outcome," but this statement is incorrect. As described, and per the amended study protocol, a gatekeeper analysis was chosen in which no additional analysis of the secondary objectives would be performed when the primary analysis was negative. We have chosen this design for several reasons. First, endoxifen is considered responsible for the pharmacologic effect of tamoxifen, and only $42.3 \%$ of variation of endoxifen levels can be explained by CYP2D6 phenotype. ${ }^{4}$ With this knowledge, it is reasonable to first investigate the variable closest to the pharmacologic effect.

In addition, it is generally accepted that a prerequisite for therapeutic drug monitoring is that there is a proven relationship between drug levels and clinical outcome, for which we believe there is currently insufficient evidence in the case of tamoxifen. In this metastatic setting, Neven et al ${ }^{9}$ showed that 21 of 51 patients in 
the lowest range of endoxifen levels ( 0.7 to $6.9 \mathrm{ng} / \mathrm{mL}$ ) had partial responses (42\%), which was not statistically significantly different from patients with higher endoxifen levels (42\% to $49 \% ; P=.56$ ). ${ }^{9}$ Fifteen of these 21 patients had endoxifen concentrations less than the $5.97 \mathrm{ng} / \mathrm{mL}$ threshold. ${ }^{10}$ This is in line with our study results: endoxifen concentrations were not associated with relapse-free survival during only-tamoxifen treatment (HR, 1.538; 95\% Cl, 0.719 to 3.290; $P=.267$ ). Although the hypothesis of an endoxifen threshold is appealing, we should consider the possibility that $20 \mathrm{mg}$ of tamoxifen per day is actually an overdose, leading to oversaturation at the level of the estrogen receptor. This hypothesis led to the initiation of a study in which the efficacy of adjuvant tamoxifen at a lower dose was tested in patients who had ductal carcinoma in situ or lobular carcinoma in situ. Recently, DeCensi et a ${ }^{11}$ showed that $5 \mathrm{mg}$ of tamoxifen once daily for 3 years halves the incidence of recurrence (HR, 0.48) compared with placebo. ${ }^{11}$ On the basis of these results, we doubt the validity of the endoxifen thresholds and thus cannot agree with therapeutic drug monitoring for tamoxifen at present.

Pharmacogenetic biomarkers to improve the efficacy and safety of therapy are of great value. However, because the majority of biomarker studies failed to be reproduced, we emphasize the necessity for replication before implementation in clinical care. Therefore, we encourage researchers and scientific journals to publish positive and negative findings of well-designed pharmacogenetic studies that can build a solid evidence base for the use of pharmacogenetic biomarkers in the treatment of patients.

Henk-Jan Guchelaar, PharmD, PhD and Anabel Sanchez-
Spitman, PharmD
Leiden University Medical Center, Leiden, the Netherlands

Vincent Dezentjé, MD, PhD

Netherlands Cancer Institute/Antoni van Leeuwenhoek, Amsterdam, the Netherlands

Stefan Böhringer, PhD, MD and Jesse Swen, PharmD, PhD

Leiden University Medical Center, Leiden, the Netherlands

Patrick Neven, MD, PhD

University Hospitals Leuven, Leuven, Belgium
Hans Gelderblom, MD, PhD

Leiden University Medical Center, Leiden, the Netherlands

AUTHORS' DISCLOSURES OF POTENTIAL CONFLICTS OF INTEREST AND DATA AVAILABILITY STATEMENT

Disclosures provided by the authors and data availability statement (if applicable) are available with this article at DOI https://doi.org/10.1200/ JCO.19.00932

\section{ACKNOWLEDGMENT}

The authors thank Roche for providing the Amplichip P450 test, Integraal Kankercentum Nederland for data management, and the support from the Zoleon (Stichting Oncologie HollandWest) Project 09.09 grant.

\section{REFERENCES}

1. Braal CL, Beijnen JH, Koolen SLW: Relevance of endoxifen concentrations: Absence of evidence is not evidence of absence. J Clin Oncol 37: 1980-1981, 2019

2. Brauch H, Schroth W, Mürdter T, et al: Tamoxifen pharmacogenetics and metabolism: The same is not the same. J Clin Oncol 37:1981-1982, 2019

3. Goetz MP, Suman VJ, Nakamura Y, et al: Tamoxifen metabolism and breast cancer recurrence: A question unanswered by CYPTAM. J Clin Oncol 37:1982-1984, 2019

4. Sanchez-Spitman A, Dezentjé V, Swen J, et al: Tamoxifen pharmacogenetics and metabolism: Results from the prospective CYPTAM study. J Clin Oncol 37:636-646, 2019

5. Goetz MP, Rae JM, Suman VJ, et al: Pharmacogenetics of tamoxifen biotransformation is associated with clinical outcomes of efficacy and hot flashes. J Clin Oncol 23:9312-9318, 2005

6. Gonzalez-Santiago S, Zárate R, Haba-Rodríguez J, et al: CYP2D6*4 polymorphism as blood predictive biomarker of breast cancer relapse in patients receiving adjuvant tamoxifen. J Clin Oncol 25:590-590, 2007

7. Province MA, Goetz MP, Brauch $\mathrm{H}$, et al: CYP2D6 genotype and adjuvant tamoxifen: Meta-analysis of heterogeneous study populations. Clin Pharmacol Ther 95:216-227, 2014

8. Berry DA: CYP2D6 genotype and adjuvant tamoxifen. Clin Pharmacol Ther 96:138-140, 2014

9. Neven P, Jongen L, Lintermans A, et al: Tamoxifen metabolism and efficacy in breast cancer: A prospective multicenter trial. Clin Cancer Res 24: 2312-2318, 2018

10. Madlensky L, Natarajan L, Tchu S, et al: Tamoxifen metabolite concentrations, CYP2D6 genotype, and breast cancer outcomes. Clin Pharmacol Ther 89:718-725, 2011

11. DeCensi A, Puntoni M, Guerrieri GA, et al: A randomized placebo controlled phase III trial of low dose tamoxifen for the prevention of recurrence in women with operated hormone sensitive breast ductal or lobular carcinoma in situ. Cancer Res 79, 2019 (abstr GS3-01)

DOI: https://doi.org/10.1200/JC0.19.00932; Published at jco.org on June 18, 2019. 


\section{Reply to C.L. Braal et al, H. Brauch et al, and M.P. Goetz et al}

The following represents disclosure information provided by authors of this manuscript. All relationships are considered compensated. Relationships are self-held unless noted. I = Immediate Family Member, Inst = My Institution. Relationships may not relate to the subject matter of this manuscript. For more information about ASCO's conflict of interest policy, please refer to www.asco.org/rwc or ascopubs.org/jco/site/ifc.

Patrick Neven

Consulting or Advisory Role: Novartis (Inst), Lilly (Inst), Pfizer (Inst), Ipsen (Inst) Travel, Accommodations, Expenses: Pfizer, Roche
Hans Gelderblom

Patents, Royalties, Other Intellectual Property: Amgen (Inst), BoehringerIngelheim (Inst), Pfizer (Inst), Novartis (Inst), Pharmamar (Inst), Daiichi (Inst), Five Prime (Inst)

No other potential conflicts of interest were reported 